Voix et Images

\title{
Paul-Marie Lapointe et la question de l'Amérique
}

\section{Pierre Nepveu}

Volume 17, numéro 3 (51), printemps 1992

Paul-Marie Lapointe

URI : https://id.erudit.org/iderudit/200977ar

DOI : https://doi.org/10.7202/200977ar

Aller au sommaire du numéro

\section{Éditeur(s)}

Université du Québec à Montréal

\section{ISSN}

0318-9201 (imprimé)

1705-933X (numérique)

Découvrir la revue

\section{Citer cet article}

Nepveu, P. (1992). Paul-Marie Lapointe et la question de l'Amérique. Voix et Images, 17(3), 435-445. https://doi.org/10.7202/200977ar d'utilisation que vous pouvez consulter en ligne.

https://apropos.erudit.org/fr/usagers/politique-dutilisation/ 


\title{
Paul-Marie Lapointe et la question de l'Amérique
}

\author{
Pierre Nepveu, Université de Montréal
}

\begin{abstract}
La poésie de Paul-Marie Lapointe, principalement dans Choix de poèmes. Arbres et Pour les âmes, n'est pas une "célébration de l'Amérique ". La forme même de cette poésie donne à entendre et à sentir l'Amérique comme un recueil de traces, dans une tension constante entre l'acte d'babiter, où domine l'impulsion érotique, et une mémoire douloureuse qui ne se déploie jamais en récit, mais plutôt en fragments d'un passé enfoui. Du point de vue québécois, l'Amérique est une "chambre d'échos", une mélancolie persistante pourtant en-partie surmontée par l'effort bumain d'babiter toute la Terre et de recréer sans cesse le monde.
\end{abstract}

Le mot * Ámérique. n'apparaît nulle part dans Le Réel absolu. PaulMarie Lapointe n'est pas un * chantre * du continent, il n'est pas de son propos de faire écho à l'invocation d'un Louis Fréchette: *Amérique! salut à toi, beau sol natal ${ }^{1}$." L'Amérique n'est pas pour lui un "problème ", au sens où il s'agirait d'interroger notre appartenance tant de fois niée ou différée à ce continent. Il est vrai que ce thème est omniprésent dans la poésie québécoise à l'époque où s'écrivent Choix de poèmes. Arbres et Pour les âmes, que ce soit chez Michel van Schendel ou Paul Chamberland, chez Gaston Miron ou Gatien Lapointe, voire chez Fernand Ouellette. Lapointe lui-même y fait clairement écho dans ce texte tant de fois cité où il place son projet poétique sous le signe du jazz et se réclame par là d'^une nouvelle forme de lyrisme, une forme nord-américaine ${ }^{2}$.

1. Louis Fréchette, La Légende d'un peuple, Trois-Rivières, Ecrits des Forges, 1989, p. 30.

2. -Notes pour une poétique contemporaine, dans Guy Robert, Littérature du Québec. Poésie actuelle, Montréal, Librairie Déom, 1970, p. 203. 
Le mot "Amérique" résonne toujours dans notre poésie comme un grand cri, un appel qui devient vite déchirant tant il exprime une sorte de convoitise non rassasiée, la proximité d'un impossible, comme dans ces derniers mots adressés au Gilles de la "Suite fraternelle. de Jacques Brault: "tu es la promesse qui gonfle les collines de mon pays ma femme ma patrie étendue au flanc de l'Amérique ${ }^{3}$. Ces mots disent, on ne peut mieux, "la profondeur de la mélancolie continentale qui attend toujours sa solution dans l'âme québécoise ${ }^{4}$. À partir de cette mélancolie, tous les malentendus sont possibles, y compris celui qui concerne le terme même d' Amérique ", confisqué par l'épopée yankee et repris à son compte (ce qui ne nous arrange guère) par la France de telle manière que nous ne pouvons toujours être qu'à côté de l'Amérique, voués à n'être que "cette Amérique en quête d'Amérique dont a parlé Jean Morisset ${ }^{5}$. Le problème reste toujours le même: de quelle Amérique parlons-nous et surtout, que cherchons-nous à travers ce mot: une réalisation enfin totale de nous-mêmes ou une fuite dans un pur imaginaire dépourvu d'expérience concrète et d'historicité? Incapables de répondre, nous avons le plus souvent adopté, jusqu'à aujourd'hui, la stratégie de l'identification à un personnage type, à un héros plus grand que nature (même dans la souffrance) détenant à lui seul la clé de l'Amérique: le Voyageur, l'Indien, le Nègre (blanc), le Cowboy, le Poète beat, la Star hollywoodienne. Ce n'est pas par hasard que le plus beau livre qui se soit écrit au Québec sur l'Amérique (au sens des États-Unis) soit le Melville de Victor-Lévy Beaulieu, livre où se dit, avec toute la passion et toute la lucidité propres à Beaulieu, cet immense désir mimétique qui nous travaille lorsque nous pensons "Amérique et lorsque nous allons au-delà d'une vague référence au *tellurisme " de ce continent ${ }^{6}$.

Ce qui frappe lorsque l'on relit les grands poèmes du Réel absolu, c'est précisément que toutes nos références archi-connues à l'Amérique (depuis sa définition territoriale plus ou moins restrictive ou généreuse jusqu'à cette galerie de personnages mythiques nous initiant illusoirement au vaste continent), tous les repères habituels donnant le change sur notre "américanité" se trouvent brouillés: pas forcément abolis,

3. Jacques Brault, Poèmes 1, Saint-Lambert, Éditions du Noroît/La Table rase, 1986, p. 55.

4. La formule est de Jean Larose, .Le cheval du réel, L'Amour du pauvre, Montréal, Boréal, 1991, p. 87.

5. Jean Morisset, Québec amériquain/Québec américain ou la poursuite de la différence invisible! ., Possibles, vol. VIII, n 4, été 1984, p. 26.

6. On pourrait en dire autant du film capital de Jacques Godbout, Alias Will James, ou le mimétisme québécois d'une certaine Amérique western atteint à une grandeur mythique (voir à ce sujet l'analyse de Jean Larose, op. cit., p. 87-100). 
mais disposés de telle sorte qu'il faut tout reprendre, tout imaginer à neuf. La poésie de Lapointe ne nomme pas l'Amérique, elle la donne à sentir et à penser sans jamais en arrêter la définition, sans la fixer dans une figure ou un lieu précis. Même dans son texte programmatique sur le jazz, Lapointe s'empressait d'ailleurs de préciser que son lyrisme nord-américain ne pouvait se concevoir sans un recours constant aux "vieilles cultures, européennes et chrétiennes " autant qu'aux traditions africaines, asiatiques ou amérindiennes. John Coltrane ou Miles Davis ne proposent pas des alternatives ou des antidotes à une culture classique supposément morte, ils ne sont pas le contraire de Vivaldi, mais plutôt son prolongement, comme ils font écho aux chants douloureux du prophète Jérémie ou de tel troubadour en mal d'amour. Obnubilés par le ton souvent "sauvage" du Vierge incendié, on a sous-estimé l'humanisme foncier de Lapointe, un humanisme explicite qui ne se prive pas de parler de "la grandeur de l'homme* (sans en négliger les petitesses et les misères) ni d'un héritage à transmettre.

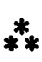

L'horizon américain se dessine, dans Le Réel absolu, à l'intérieur d'un thème plus vaste qui est en fait le seul qu'ait jamais traité Lapointe: "la création du monde et sa fin", comme le disent les premiers vers de "Blues* (p. 249)7. Il ne s'agit toujours que de cela et en ce sens, l'Amérique ne fait qu'activer, avec un peu plus d'intensité, ce rapport aux limites, naissance et mort, origine et perte, qui détermine toute existence, en même temps qu'elle illustre tout ce qu'il y a de plus enivrant et de plus dangereux dans l'idée même de modernité telle qu'elle s'est imposée depuis la Renaissance jusqu'à Rimbaud: Ces héritiers (critiques) de Walt Whitman qu'ont été Hart Crane et William - Carlos Williams, deux des poètes qui se sont le plus directement mesurés à l'Amérique, avaient déjà très bien compris, chacun à sa manière, qu'il est facile, trop facile, de *chanter * après Whitman ce continent et que, malgré sa grandeur, le poète de Leaves of Grass ne saurait être un modèle à imiter. Au-delà de celui qui demeure le poète archétypal de l'Amérique, on ne peut plus écrire qu'à rebours de Whitman. Toute la poétique de Williams constitue une dénonciation explicite de celle de Whitman, jugée informe ( a magnificent failure",

7. Toutes les références à l'œuvre de Lapointe renvoient à l'édition du Réel absolu, Montréal, 'L'Hexagone, coll. *Rétrospectives •, 1971. 
écrit Williams ${ }^{8}$ ), et par là inapte à saisir l'expérience concrète de ce continent. Et Crane ne dit toute sa fascination et son amour pour lui, dans The Bridge (voir la section intitulée " Cap Hatteras"), que dans un mouvement endiablé qui finit par dire le contraire de ce que disait Leaves of Grass: l'épopée yankee et sa conquête de l'ouest, si enivrantes soient-elles, illustrent en même temps tout ce qu'il y a de plus catastrophique dans la modernité, ce déploiement fiévreux d'une énergie vitale qui méconnaît le mal et la mort, ce qui finit par créer des hommes et tout un peuple d'aveugles, qui ne savent pas ce qu'ils sont ni ce qu'ils font, traînant en eux-mêmes une âme morte, un cadavre oublié.

Malgré l'absence d'un thème national ou explicitement continental chez Grandbois ${ }^{9}$, il y a chez lui du Whitman, comme il y en aura plus tard d'une façon bien plus nette chez un Gatien Lapointe et comme on en retrouve des échos dans la poésie d'un Pablo Neruda. En fait, c'est une perpétuelle tendance des diverses poésies des Amériques à chercher l'essence du continent *dans sa masse physique, dans son statut cosmologique", comme l'a montré Jaime Concha dans un article consacré à Neruda ${ }^{10}$. Borduas lui-même, dans Refus global, ne parlait-il pas de ce "petit peuple, que nous sommes, "au nord de l'immense Amérique au corps sémillant de la jeunesse au cœur d'or *? Dans cette saisie à bras-le-corps d'une nature pléthorique et illimitée, l'Amérique peut apparaître aux plus lucides comme l'«essor des modernes " dont parlait Hart Crane, au sens où elle rend manifeste l'aveuglement même qui caractérise cet essor, ne pouvant désormais qu'être le lieu par excellence où Satan et la mort (et aussi, curieusement, une certaine vieillesse, mauvaise et prématurée) ressurgissent avec une virulence particulière.

La poésie de Paul-Marie Lapointe a ceci de particulier qu'elle reproduit comme en filigrane, sur le mode de l'allusion réitérée, la trajectoire propre à tous ceux qui "découvrent" l'Amérique, ou qui y sont simplement venus pour recommencer une nouvelle vie. Le Vierge incendié n'est sûrement pas américain au sens d'un territoire, d'un paysage,

8. The Selected Letters of William Carlos Williams, avec une introduction de John C. Thirlwall, New York, McDowell and Obolensky, 1957, p. 135.

9. Cela est vrai davantage sur le plan de la forme que du contenu, car c'est bien davantage dans un livre comme Né à Québec que Grandbois se mesure, comme dirait Gaston Miron, à l'Amérique. Comme presque toujours (jusqu'à Jacques Poulin), la véritable poésie de l'Amérique est pour nous narrative, elle prend la forme de la pérégrination, du pèlerinage, de la remémoration.

10. Jaime Concha; - Neruda et son continent natal., Etudes littéraires, vol. VIII, $\mathrm{n}^{\circ} 1$, avril 1975, p. 135. 
d'une faune ou d'une population spécifiques. C'est plutôt dans le mouvement et l'esprit qui caractérisent ce livre, dans sa jeunesse débridée proclamant la nécessité de partir, de se détacher du "passé / qui mérite de pourrir derrière soi * (p. 122) et d'aller vers le scintillement du * futur de cristal. (p. 82), que l'on peut parler de l'ébauche d'une américanité qui deviendra explicite dans les recueils du début des années soixante.

Mais ce mouvement qui devrait être de pure libération est loin d'être simple. Tout le grand cirque exotique et primitiviste du Vierge incendié, cette panoplie de cannibales et de caïmans, de huttes indigènes, de nègres et de créoles qui mordent, cela ne va pas sans un lexique sans doute largement livresque du "vieux monde" (sans compter un certain bazar africain). Non seulement voit-on apparaître ici et là un empereur, un roi, un duc ou une comtesse, mais ce qui est encore plus significatif, le langage du jeune Lapointe porte partout les traces d'un code français purement emprunté, tout un monde de boulevards et d'autocars, de carrosses lanternés et de cabs, de bergers, de gosses et de gendarmes. Quand on sait que plus tard.Lapointe fera un inventaire systématique des espèces d'arbres de cette partie nordique dù continent, on mèsure à quel point la recherche d'une «forme nord-américaine " a dû passer par un exercice de nomination, par la récupération d'un champ référentiel absent ou inaccessible au départ.

Le Vierge incendié a beau se terminer par une création du monde qui dit que "toutes les routes sont ouvertes* (p. 117) et que "tous les rivages sont loin de moi * (p. 124), la récurrence de termes comme "pourrir", "vieux" et "mort " indique assez que l'adolescent rageur qui fait éclater le monde et le langage regarde tout autant en arrière qu'en avant: "mais maintenant qu'il ne me reste plus qu'à regarder en arrière avenir serein du suicide" (p. 66). Et plus loin: "tant de murs d'en arrière à démolir/à reconstruire demain. (p. 121). Tout Le Vierge incendié voudrait en finir avec le vieux monde et produire «l'horizon neuf " évoqué dès les premières pages. Mais ce qui est en arrière est aussi une figure du futur. Le décrochage par rapport au monde civilisé confine à un curieux retour des choses: "Lamentez-vous cors de France j'ai perdu mon rond cerveau le seul instinct subsiste dans la vitesse" (p. 93). "Instinct ", "vitesse": comment ne pas reconnaître là deux des qualités si souvent revendiquées et mises en œuvre par l'Amérique, et devenues tant de fois la raison même d'une perdition? :[J]e veux un destin neuf corde neuve et la mort au bout ", poursuit le même texte. Le nouveau sauvage qui rencontre à la fin "les yeux des iroquois. (p. 124) découvre qu'il vient d'entrer par sa révolte tous azimuts dans un monde circulaire, où le désir apparemment sans frontières finit toujours par un nouveau mur, une nouvelle ruine, une 
autre mort. Il n'y a peut-être pas de nouveau monde ou s'il y en a un, ce n'est pas par un aller simple qu'on le rencontrera, ni par une identification rapide à un mythe emprunté.

Dans la Nuit du 15 au 26 novembre 1948, Lapointe sera hanté à nouveau par l'idée de départ ("Il faut partir. [p. 142]; "Jamais depuis qu'on est parti/ On ne revient dans son pays. [p. 149]; * Il a fallu partir et qui n'est parti / Pourrira nous l'avons assassiném. [p. 157]). Mais pour aller où? Quand un Américain repart, après avoir quitté la vieille Europe, ce peut être pour aller plus loin en Amérique, c'est-à-dire chéz lui. Ce peut-être, comme Walt Whitman, pour se plonger dans le souffle sauvage du pays-continent, dans son énergie cosmique. Mais une fois constaté qu'^il n'existe point de volcan dans Québec* (p. 143), le locuteur de Lapointe paraît singulièrement dépourvu. Bien sûr, comme tant d'Américains (ou comme tant d'Européens en mal d'Amérique), il va du côté du plus "sauvage", mais cela n'est tout simplement pas sérieux, cela ressemble à une mauvaise blague: «les iroquois prétendent que je suis torturé mais je ne me suis jamais senti mieux. (p. 158). C'est moins la réapparition des Indiens qui importe ici que son registre parfaitement burlesque, comme celui du parler «sauvage ", cette révolte contre les contraintes de la langue qui a si souvent marqué les tentatives de possession symbolique de l'Amérique (jusque dans l'exploréen de Gauvreau). Non seulement le "ceci est du sauvage" (p. 157) de Lapointe est une blague, mais un des derniers textes de la Nuit finit par avouer: "On voudrait bien parler à un mur qui est devant soi mais puisque ce n'est pas vraiment un mur puisque tous les murs sont avalés et qu'on est seul dans son pays" (p. 162). Cette phrase qui demeure en suspens referme en même temps une boucle: bien des murs se sont effondrés ou ont été dévorés dans $L e$ Vierge incendié, mais au bout du compte c'est la solitude et la petitesse québécoise qui l'emportent.

\section{**}

Avec Cboix de poèmes. Arbres, la perspective change radicalement. D'abord, la référence continentale devient explicite, elle n'est plus seulement le sous-entendu d'une insurrection contre les formes et le langage. S'il doit y avoir cette "création du monde et sa fin" qu'exige l'existence humaine, ce doit être par la convocation de toutes les harmoniques d'une vaste musique continéntale. Mais il n'y a pas de privilège mythique accordé à l'Amérique: aventure québécoise, américaine et planétaire, ces niveaux d'intégration de l'expérience ne peuvent évidemment être isolés l'un de l'autre. Pas plus que le grand hymne 
aux *arbres* du continent ne peut être isolé du chant érotique au corps de l'amante ou de la plainte douloureuse de "Soyez tristes". Éros et douleur, amour et terreur: telles sont les données fondamentales de cette habitation du monde, de cette possession du réel perçu à partir d'un environnement qui a son caractère propre: géographie, saisons, villages et villes. Nous sommes indubitablement en Amérique: toutefois, cette référence à un espace donné est loin de caractériser avec suffisamment de précision l'entreprise poétique de Lapointe.

Il me semble que deux éléments particuliers doivent être pris en compte. Le premier concerne l'invention américaine dans son ensemble et touche le rapport à l'histoire. Le second (j'y viendrai en fin de parcours) concerne le point de vue particulier du Québec sur l'Amérique.

Le premier point est inséparable de la forme même qu'adopte la poésie de Lapointe à partir d'“Arbres": la pratique du fragment, de la juxtaposition de notations libres à l'intérieur d'un champ thématique assez large. Cette forme permet en effet de dire l'Amérique non pas sur le mode d'une quête épique, ni d'une genèse mythique, mais comme un recueil d'expériences, de sensations travaillées par des bribes de mémoire. On peut dire de Lapointe ce que Simon Harel a écrit à propos du Volkswagen Blues de Jacques Poulin, qu'il décrit al'envers


les poèmes qui suivent ne font pas cela à l'intérieur du modèle d'une quête, d'un parcours, mais plutôt d'une sorte de chant rituel, rempli de répétitions, et où ressurgissent sans cesse des morceaux du rêve américain, beau rêve d'un nouveau monde et d'une habitation neuve, mais aussi cauchemar de la violence et de la mort.

Un des mots-clés de la poésie de Lapointe à partir d' Arbres" est le mot " terre " et il apparaît dès le début du poème: * racines de la pluie et du beau temps terre animée. (p. 171). Cette poésie du terrestre qui dit le « réel absolu * de la fondation et du présent (corps et paysage traversés par un même érotisme) ne cesse de faire écho à un récit que tout habitant de cette partie boréale du Nouveau monde reconnaît aussitôt comme sien. Ainsi, dès les premières séquences d' Arbres" apparaissent ces "pins roulés dans leur neige [qui] traversent les années mâts fiers voiles tendues sans remords et sans larmes équipages armés" (p. 171), et un peu plus loin, le "bouleau à l'écorce fendant l'eau des fleuves. (p. 172). Le poème fera ensuite une pause pour dire ceci:

11. Simon Harel, Le Voleur de parcours, Longueuil, Le Préambule, coll. .L'Univers des discours :, 1989, p. 168. 
arbre

arbre pour l'arbre et le Huron

arbre pour le chasseur et la hache

arbre pour la sirène et le blé le cargo le cheval

(p. 173)

Entre l'évocation d'une traversée en voilier dont les intentions sont visiblement conquérantes, celle du canot d'écorce et celle du Huron, de la forêt, du blé, etc., le poème donne à l'Amérique la couleur du mémorable. Cette mémoire, toutefois, semble dire du même coup la précarité de la notion d'histoire sur ce continent. Le passé se donne par morceaux à l'intérieur d'une sorte de transe cosmique, nulle part plus sensible que dans cette magnifique séquence où les conifères apparaissent successivement comme des "dons quichottes, des clairons droits foudroyants le ciel et des arêtes de poissons verticaux dévorés par l'oiseau • (p. 172). Paysage halluciné, «terre animée " par la passion, où le temps historique semble se défaire à mesure dans la présence d'un concret mouvant, en constante métamorphose.

Sur une de ses faces, la dimension américaine de la poésie de Lapointe exprime le rêve d'une présence au monde qui serait purement heureuse, parce qu'elle se serait purifiée du poids de l'histoire: corps-paysage, paysage-corps. Heureuse circulation entre "les caresses mauves et l'arbre sucré de nos os.. Tout ce qu'il y a de naïf et d'utopique dans ce rêve, la poésie de Lapointe l'assume: l'idée d'Amérique, après tout, est elle-même une idée naïve, celle d'une (re)création à partir de presque rien, celle de l'absolument neuf, partagée par tant de "petits hommes " en mal de recommencement et d'habitation heureuse du monde, rêvant de mettre leur famille à l'abri des calamités, se proposant simplement (comme si c'était simple) d'«aimer la terre/ étrangère appropriée. (p. 254). Cette naïveté n'a d'égale que la belle candeur de l'amant qui parle aux *seins" de son amante et qui croit les entendre lui répondre: "nous sommes nus nous sommes nus / pour habiller les jours. (p. 185). Sans cette naïveté, semble pourtant dire Lapointe, que serions-nous?

Comme passion érotique et cosmologique, il se pourrait que l'Amérique soit en ce sens la plus belle tentative de concrétisation de l'idée de "réel absolu * énoncée par Novalis, et il est significatif qu'un Emerson par exemple, ait parlé de l'espace américain comme d'un "gigantesque poème" étendu sous ses yeux. "L'Amérique comme un poème : comment ne pas entendre dans une telle formule l'écho d'une américanité flamboyante qui cherche à se sauver de la mort par "l'espace ", ce mot si prestigieux, si profondément magique et trompeur, et tellement américain en même temps? Ici encore, c'est Whitman qui a 
posé les paramètres de cette "poésie de l'espace", dans des poèmes comme "Starting from Paumanok ", avec cet élan si caractéristique qui va de Long Island et Manhättan vers la totalité du continent.

Le récit de fondation (et donc aussi de destruction) qui affleure par bribes dans les longs poèmes de Lapointe est au contraire inséparable d'une poésie de l'Amérique en tant que lieu. Non pas l'élan à travers l'espace, l'appel d'un au-delà, mais plutôt le désir simple d'une "résidence sur la terre", désir marqué par ces si nombreuses références aux lieux d'habitation (villes et villages, maisons et buildings) qui parsèment les poèmes. L'arbre lui-même est "croix de construction" et il est significatif qu'aux *équipages armés. évoquant la traversée fondatrice, súccèdent aussitôt les * pins des calmes armoires et des maisons pauvres/bois de table et de lit" (p. 171), de la même façon aussi que le "bois d'avirons" devient immédiatement celui des "dormants et des poutres".

La distinction entre "espace " et «lieu " est fondamentale pour saisir l'américanité de Lapointe (comme elle le serait pour définir en quoi un Williams Carlos Williams révise et corrige l'américanité d'un Whitman). L'espace permet de se sauver, ou de croire que l'on se sauve (dans les deux sens d'une fuite et d'un salut). Le lieu est éminemment vulnérable: son ici-maintenant constitue à la fois le projet le plus noble et le plus fragile. L'habitation est chaleur, proximité des choses et des corps. Mais en même temps, elle est la possibilité d'entendre tous les bruits, mêmes les plus anciens que l'on croyait enfouis. La maison du réel craque dans ses poutres, on ne peut s'enlever de la tête qu'elle est hantée. Il y un mouvement spontané, naturel, chez Lapointe, qui fait ainsi glisser sans cesse le poème du plaisir d'habiter vers une menace sourde ou une mémoire douloureuse. Même les métaphores puisées dans l'expérience américaine et que l'on pourrait qualifier d'heureuses portent en elles cette sourde menace: la séquence érotique qui ouvre "Gravitations " ("le corps se divise pour le plaisir/et la satisfaction [...] ainsi le corps se tend/il est l'arc de l'indienne / sa plus tendre peau / le tam-tam le plus sonore. [p. 214]) fait aussitôt ressurgir, à partir de la métaphore indienne, le développement suivant:

nous écoutons passer les ancêtres sous la terre

leurs attelages

et leurs convois de plumes

(guerriers occis. ossements d'une faim sans maïs, la neige pousse, blanche comme un peuple. saisons arquebuse invasion sans terre)

(p. 214) 
Lapointe parvient de cette manière à évoquer à la fois l'Éros indien qui a toujours été au cœur du rapport symbolique à l'Amérique ${ }^{12}$, et ce que cet Éros comporte de mémoire hantée, de passé mal enterré, à fleur de peau et de terre.

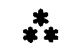

J'en viens, tardivement; au deuxième aspect de la spécificité américaine de Lapointe, celui qui concerne la dimension proprement québécoise. On connaît ce passage capital de "Soyez tristes":
ce continent me trahissait
j'étais prisonnier de ses pores
prisonnier de ses blessures
plaie quotidienne d'un espoir
ce continent me trahissait ce pays ce cercueil
par le rocher la sentinelle
par la matraque et la plume
et la hanche portant sa fillette scalpée
les amours fleurissaient dans le fumier
pivoines de la folie
(p. 198)

D'un certain point de vue pas forcément québécois, il est vrai que l'Amérique peut toujours apparaître comme une blessure et une trahison: une promesse non tenue, un vaste espace confinant ironiquement à la myopie. Mais quand Lapointe écrit *ce pays ce cercueil . ou, dans le même poème, "pleurez ce peuple est inutile", il dit davantage. Il y a une souffrance de l'Amérique, une conscience de l'enfermement et de la mort, qui ne peut être que québécoise, tout simplement parce que nous sommes un morceau meurtri de l'Amérique, et que cette blessure ne peut être identique à celle d'un Américain ni à celle d'un Amérindien. Ce constat, Lapointe le fait sans masochisme et lorsqu'il affirme: "nous sommes de petits catholiques tristes" (p. 254), il le fait à l'intérieur d'une séquence de "Blues* qui commence par: : mexique de mon amour terre lointaine / nous te posséderons. (p. 254). Désir d'une fraternité continéntale (au-delà de l'«épée " états-unienne), d'une expansion amoureuse qui permettrait de

12. Une des plus anciennes représentations allégoriques de l'Amérique, aux côtés des autres continents, fut celle d'une femme nue coiffée de plumes: on la retrouve en Europe dès la deuxième moitié du XVle siècle, et on en trouve des échos chez Hart Crane, dans la figure de Pocahontas, l'Indienne de Virginie perçue comme la figure même d'une appropriation érotique du continent. 
surmonter notre position étriquée, marginale et inessentielle sur ce continent.

Le point de vue typiquement états-uniens sur l'Amérique est celui d'un rêve tourné au cauchemar, celui d'une décadence à partir d'une origine pourtant prometteuse, celui d'une grande marche vers le progrès, la civilisation, le Bien, devenue une course vers le succès et le profit. Mais le point de vue québécois fait plutôt de l'Amérique une chambre d'échos: une sorte de souvenir persistant, une présence obsédante et pourtant jamais complètement réalisée, une hantise qui nous met à part et qui pourtant n'empêche pas que nous soyons bel et bien, pour une très vaste majorité, des Blancs d'Amérique. Nous pouvons bien jouer aux sauvages, revendiquer une sorte d'amérindianité symbolique qui fait notre affaire, comme nous avons revendiqué, surtout à l'époque de Pour les âmes, une négritude de circonstance: mais cela ne convainc guère, et moins que jamais à une époque où le "vrai* Amérindien et le "vrai * Noir se dressent devant nous et nous interpellent de l'intérieur même de notre lieu.

Lapointe touche beaucoup plus juste en saisissant les inflexions de notre mélancolie américaine, qui nous fait vivre ce continent comme une perpétuelle et inguérissable blessure, comme un rituel commémoratif qui vient toujours marquer du sceau de la mort les élans érotiques les plus beaux. En ce sens, nous n'avons pas à nous dire ni à nous vouloir américains: parce que nous portons l'Amérique en nous, dans toutes les étapes de son développement, mais aussi parce que l'"Amérique. ne saurait nous fournir la clé ultime de notre identité. Il y a toujours un au-delà de l'Amérique, comme il y a un audelà du Québec: il y a ce monde des "petits hommes", où le mot "terre" retrouve toute sa noblesse, où il n'est pas synonyme de territoire, et où il n'y a que cette expérience humaine par excellence: «la création du monde et sa fin. 